\title{
Un vaste chantier de collections
}

MétaLmorphoses et chantiers de collections : faire de la contrainte une opportunité

A vast collection site. MetaLmorphoses and collections projects: turning

constraints into opportunities

\section{Dominique Antérion}

\section{OpenEdition}

Journals

Édition électronique

URL : https://journals.openedition.org/artefact/2358

DOI : 10.4000/artefact.2358

ISSN : 2606-9245

Éditeur:

Association Artefact. Techniques histoire et sciences humaines, Presses universitaires du Midi

\section{Édition imprimée}

Date de publication : 6 décembre 2018

Pagination : $314-320$

ISBN : 978-2-8107-0595-5

ISSN : 2273-0753

Référence électronique

Dominique Antérion, « Un vaste chantier de collections », Artefact [En ligne], 8 | 2018, mis en ligne le 21 juin 2019, consulté le 21 septembre 2021. URL : http://journals.openedition.org/artefact/2358 ; DOI : https://doi.org/10.4000/artefact.2358

\section{c) (1) $\ominus$}

Artefact, Techniques, histoire et sciences humaines est mise à disposition selon les termes de la Licence Creative Commons Attribution - Pas d'Utilisation Commerciale - Pas de Modification 4.0 International. 


\title{
Un vaste chantier de collections
}

\author{
MétaLmorphoses et chantiers de collections : \\ faire de la contrainte une opportunité
}

Dominique Antérion

\section{Résumé}

Grandement impactées par MétaLmorphoses, gigantesque chantier de restructuration de l'Hôtel de la Monnaie (Paris 6e), les collections historiques de la Monnaie de Paris ont vécu entre 2012 et 2018 des années difficiles ! Entre sécurisation, externalisation, obligation de récolement, création du nouveau musée et projet de nouveau médaillier ce sont au final plus de 140000 objets qui ont été concernés par cette opération d'envergure. Néanmoins, les contraintes ont pu parfois devenir des opportunités. Outre le sens de l'adaptation des équipes, l'ouverture du nouveau musée du 11 Conti - Monnaie de Paris, l'implantation du nouveau médaillier, la poursuite du récolement décennal, témoignent de ce que l'on peut considérer comme l'un des plus vastes chantiers de collections menés en France ces dix dernières années.

\section{Mots-clés}

collection, musée, monnaie, médaille, Monnaie de Paris, conservation, restauration, récolement

99 Dominique Antérion, « Un vaste chantier de collections », Artefact, 8, 2018, p. 314320. 


\section{$A$ vast collection site}

MetaLmorphoses and collections projects: turning constraints into opportunities

\section{Abstract}

From 2012 to 2018, the Mint historical collections have been strongly impacted by MetaLmorphoses, a massive rehabilitation project for the restructuring of the building. Between the securing and the outsourcing issues, the inventory checking duty, the opening of a new museum and the incrementation of a new medal cabinet, more than 140000 items were part of this wide-range operation. However, some constraints have been turned into opportunities. In addition to the team's adaptation skills, the opening of a new museum, the incrementation of a new medal cabinet and the continuation of the ten-year inventory checking plan tend to prove that it is one of the largest collection works implemented in France over the last ten years.

\section{Keywords}

collection, museum, medal, Monnaie de Paris, conservation, restauration, inventory checking 
$\mathrm{L}$ e musée du 11 Conti - Monnaie de Paris, ouvert à grands renforts médiatiques en septembre 2017, ne doit pas être l'arbre qui cache la forêt. Héritées de l'ancienne Monnaie des Médailles, de la Monnaie des Espèces, et de la Cour souveraine des Monnaies, les collections historiques de la Monnaie de Paris brillent par leur importance historique, qualitative et quantitative qui les classe parmi les collections numismatiques (au sens large du terme) de référence. Si le parcours permanent du " 11 Conti " expose au public un peu moins de 2000 objets répartis en huit sections, les collections en réserves sont estimées pour leur part à plus de 140000 objets ! Bien davantage à n'en pas douter, si l'on en juge par les chiffres du récolement décennal qui dépassent déjà les 110000 items alors qu'il ne concerne qu'au mieux un tiers des espaces de réserves visités.

\section{MétaLmorphoser l'Hôtel de la Monnaie}

Le récolement est une obligation légale à laquelle sont soumis les musées contrôlés et/ou labellisés " Musée de France ». L'opération - qui consiste à s'assurer que les collections sont en conformité avec les registres d'inventaires - peut être vue soit comme une contrainte, soit comme une opportunité. Clairement, le département de la conservation de la Monnaie de Paris a opté pour le second angle de vue faisant de cette obligation une opération à tiroirs (sic) aux débouchés nombreux. Car au-delà du récolement, c'est à un vaste chantier de collections que la Monnaie de Paris procède depuis plusieurs années. Un chantier dû en grande partie aux effets collatéraux de MétaLmorphoses, nom donné à la restructuration quasi complète du site historique de l'Hôtel de la Monnaie qui s'est étalée de 2012 à 2017. Parmi les opérations impactant les collections, on citera la démolition des précédents musée et médaillier (pour récupération partielle des espaces), l'externalisation des collections (qui ne pouvaient demeurer in situ par manque de place et de sécurité), la création d'une réserve dédiée dans l'emprise même de l'Établissement Monétaire de Pessac, un projet de nouveau musée (dès 2009), que viendrait compléter un nouveau médaillier. Le récolement s'inscrit donc dans ce vaste mouvement de collections auquel il contribue à apporter la clarté souhaitée par le législateur. Car si tous ces mouvements d'œuvres ne sont pas sans conséquence (ni sans risques) sur les collections elles-mêmes, ils n'ont pas été également sans compliquer singulièrement la tâche du département de la conservation qui, dans le même temps, se 
devait de scénariser le futur musée du 11 Conti : choix et prélèvements des objets en accord avec le projet scientifique et culturel (PSC), prises de vues, études et analyses... Comment mener tout cela de front lorsque tout bouge, jusqu'à parfois $500 \mathrm{~km}$ de Paris?

C'est la phase 1 de MétaLmorphoses qui sonna le glas des précédents musées et du médaillier. Le musée - deuxième du nom -, installé depuis 1988 en plein cœur de l'Hôtel de la Monnaie, fut entièrement démonté en 2012, et les collections remisées au médaillier. La tâche fut d'autant plus compliquée que le transfert provisoire du médaillier fut mené pour ainsi dire dans le même temps ! Ce dernier, initialement installé en façade de l'Hôtel de la Monnaie, dans la continuité des salons du bord de l'eau, fut intégralement démonté et remonté dans une zone configurée à l'ancien musée, côté cour d'honneur. Il devait y rester dix ans avant d'être touché à son tour par la phase 2 de MétaLmorphoses, consacrée notamment à l'implantation du nouveau musée. Riche de plus de 60000 objets de petite taille (monnaies, essais, coffrets, mais aussi jetons, poids, etc.) le médaillier ${ }^{1}$ ne pouvait plus demeurer quai Conti en raison des nombreuses zones de chantier qui impactaient le site. Décision fut alors prise de l'externaliser en banlieue parisienne chez un prestataire spécialisé. Les valeurs les plus précieuses (tels le Trésor de Hué ou les "Excellence à la française ") furent quant à elles déposées dans les coffres de la Brinks. À noter que dès le démarrage de la phase 1 , les collections de médailles (70 000) et d'outillages monétaires et médaillistiques (40 000) avaient été évacuées et installées dans une réserve créée spécialement au sein de l'Établissement monétaire de Pessac. Les collections techniques les y rejoignirent, tandis que métrologie, mobiliers, tableaux, et objets d'art étaient exfiltrés dans le même temps vers deux réserves provisoires au sud de Paris.

1. Dessiné et construit en interne au début des années 1980 par les menuisiers de la Monnaie, le médaillier est constitué de bâtis en bois pouvant abriter jusqu'à soixante-douze tiroirs. Chaque bloc, soigneusement numéroté, étant consacré à une ère chronologique, géographique, ou à une section thématique. 


\section{Concevoir un nouveau musée et récoler les collections}

Rien toutefois qui ne devait empêcher les opérations liées à la conception du nouveau musée et au récolement. Réactivité et opiniâtreté furent les maîtres mots, permettant ainsi de mener les deux opérations de front, et selon un tempo en accord avec les priorités de MétaLmorphoses, dont les glissements calendaires (inhérents à des chantiers de cette ampleur) jetaient le flou sur les dates d'échéance. Pour le personnel du département de la Conservation, il fallut s'adapter et pratiquer le grand écart en tout domaine. Il fallut aussi se faire opportuniste et jouer des avancées d'un chantier pour en faire progresser d'autres. Ce fut particulièrement vrai pour la conception des multimédias (nombreux) prévus dans le musée. Des multimédias gourmands en images d’objets ; objets dont les équipes ne disposaient précisément plus. C'est là où le récolement fut de la plus grande importance. En vertu du principe qu'un objet récolé est un objet vu, on procéda dans le même temps, selon un cahier des charges strict, à la numérisation de chacun d'eux en plusieurs formats d'images (TIFF, JPG HD, JPG BD simples ou associant sur une même vue avers, revers, échelle millimétrique, et incrustation du numéro d'inventaire) (Fig. XVI, cahier couleur). Il revenait aux images JPG d'être versés dans la base de données documentaire et aux TIFF d'enrichir les multimédias que le département de la Conservation concevait alors avec les développeurs. La quasi-totalité du fonds de médailles (plus de 70 000) et d'outillages (6 700) fut ainsi photographiée par une équipe de vacataires recrutée spécialement à Pessac. Pendant ce temps, en région parisienne, un prestataire effectuait la même opération par campagnes successives. 2869 monnaies antiques et 8812 monnaies modernes (fin $\mathrm{Xv}^{\mathrm{e}}$-milieu XIX ${ }^{\mathrm{e}}$ siècle) furent ainsi récolées et photographiées, permettant l'avancement du projet légal et du projet multimédia. Tandis que des opérations de collecte s'effectuaient simultanément dans les ateliers, notamment impactés par MétaLmorphoses (fonderie, estampage, gravure, mais aussi laboratoire...), les choix s'opéraient quant aux objets à exposer dans le nouveau musée, et les prélèvements, en fonction du scénario muséographique retenu, pouvaient commen$\operatorname{cer}^{2}$. Point délicat qu'il fallait parfaitement coordonner avec le chantier :

2. C'est ainsi que furent intégrés aux collections nombre d'éléments de l'ancienne fonderie transférée elle aussi dans le cadre de MétaLmorphoses. À Pessac, une même collecte fut effectuée au niveau 
l'intégration des machines - approchant la tonne pour certaines - dans le parcours. Le couvert du futur musée et la présence d'une grue dont le démontage ne tarderait plus, imposaient de les placer dans le parcours avant même que la zone ne soit réceptionnée. Balanciers de Brézin, de Gengembre, tour à réduire, balance trieuse prirent place dix mois avant l'ouverture, impliquant des règles strictes de protection des œuvres alors situées en plein chantier.

\section{Un chantier peut en cacher un autre}

Six mois plus tard, en juin 2017, la livraison des espaces par les entreprises marquait le démarrage du chantier muséographique. Préfigurées un an auparavant, soixante-dix vitrines furent agencées et pourvues de leurs 1870 objets, scénarisés et soclés en un peu moins de six semaines. Pendant ce temps les restaurations allaient bon train : objets de laboratoire récemment collectés (Fig. XVII, cahier couleur), outillages oxydés, monnaies d'argent sulfurées, marbres encrassés. Même l'emblématique statue de la Fortune du sculpteur Louis-Philippe Mouchy (1734-1801) - qui fit l'objet d'un mécénat participatif - retrouva une seconde jeunesse. Pose des cartels, des signalétiques et autres balisages vinrent clore ce chantier auquel la commission de sécurité donna finalement son aval, autorisant ainsi son exploitation.

L'ouverture du musée au public le 30 septembre 2017 ne signifiait en rien la fin des autres chantiers. Le récolement ne devant s'interrompre, on entama dès le mois de mars 2017 celui de la collection de monnaies papiers (à cette date seule collection rapatriée intégralement in situ). Clos en décembre de la même année, il révéla une collection de 26150 items dont un impressionnant ensemble de monnaies de nécessité de la Première Guerre mondiale. Scrupuleusement reconditionné, cet ensemble fera l'objet d'une exposition dédiée dans le cadre de la clôture des commémorations du centenaire de la Grande Guerre en novembre 201833. Ou comment rebondir culturellement sur une opération purement légale!

du laboratoire, enrichissant singulièrement la collection scientifique du musée et encourageant la création d'une section à part entière du nouveau musée.

3. «14-18, la monnaie ou le troisième front », musée du 11 Conti - Monnaie de Paris, du 6 novembre 2018 au 24 février 2019. Commissariat : Dominique Antérion et Axelle Janiack / Muséographie : Nicolas Métro et Manon Sendeki / Production : Pernille Soldat. 
2018 sera également marquée par le rapatriement progressif des collections patrimoniales sur le site du quai Conti ${ }^{4}$. Avec en point d'orgue la mise en place du nouveau médaillier monétaire, implanté dans une zone en connexion avec les collections permanentes. Logiquement sécurisé, mais voulu pourtant chaleureux, le lieu accueillera, outre les monnaies et les jetons (répartis en pas moins de 1200 tiroirs récupérés du médaillier précédent), la collection de métrologie, riche notamment d'un bel ensemble de balances d'essayeurs des XVIII ${ }^{\mathrm{e}}$ et $\mathrm{XIX}^{\mathrm{e}}$ siècles, ainsi que la collection de monnaies papier. Des meubles emblématiques tel le médaillier des empereurs Napoléon Ier et Napoléon III, ou encore la vitrine "papillon ", dessinée par Christian Lacroix, compléteront l'ensemble pour en faire un lieu d'exception.

Ainsi, peu à peu rendues au public autant qu'aux spécialistes, les collections de la Monnaie de Paris reprennent place, pour leurs domaines respectifs, dans l'environnement des musées français et sur le devant de la scène numismatique. Demandes de collaborations scientifiques, emprunts d'œuvres, dons... le musée du 11 Conti-Monnaie de Paris est aujourd'hui en mesure de se refaire un nom pour le plus grand bien de ses collections et de ceux qui les animent.

4. À noter que les collections de médailles, fontes d'art, outillages et machines demeurent sanctuarisées sur le site de l'Établissement monétaire de Pessac. 